\title{
Hindsight and moving the needle forwards on rehabilitation trial design
}

\author{
Bronwen Connolly, ${ }^{1,2,3,4,5}$ Linda Denehy ${ }^{5}$
}

Hindsight: the ability to understand, after something has happened, what should have been done or what caused the event. ${ }^{1}$

The first randomised controlled trials (RCTs) of physical rehabilitation in critically ill patients in the intensive care unit (ICU) were published nearly 10 years ago demonstrating favourable results and heralding a groundswell of clinical and research activity in the field. ${ }^{23}$ Ensuing RCTs continued to investigate the peri-ICU period ${ }^{45}$ but additionally mapped the rehabilitation pathway to include post-ICU discharge on the ward $^{6}$ and community ${ }^{78}$ and across the continuum of care. ${ }^{9}$ However, this impressive body of literature has challenged a transformation in clinical practice. Recent studies have failed to show a difference in primary outcome, ${ }^{45}$ but is this truly failure of interventions to demonstrate effect or failure of intervention delivery within an appropriately designed study? Hindsight is a valuable perspective to interpret this premise. Triallists need to pre-empt advances in knowledge and understanding of the topic in order to future-proof their research. It is easy to lament what could have been done differently. Acknowledging this, these trials have contributed greatly to our current understanding of the intricacies of design, conduct and evaluation of trials of complex rehabilitation interventions in critical care.

Against this backdrop, Wright and colleagues present their Intensive versus standard physical rehabilitation therapy in the critically ill (EPICC) study, a multicentre RCT investigating intensive versus standard physical rehabilitation therapy in the ICU. ${ }^{10}$ This is the first UK trial of its kind, recruiting 308 participants to receive either intervention (target $90 \mathrm{~min}$

\footnotetext{
${ }^{1}$ Lane Fox Clinical Respiratory Physiology Research Centre, London, UK

${ }^{2}$ NIHR Biomedical Research Centre, Guy's and St. Thomas' NHS Foundation, London, UK

${ }^{3}$ King's College London, London, UK

${ }^{4}$ Centre for Human and Aerospace Physiological Sciences, King's College London, London, UK ${ }^{5}$ Department of Physiotherapy, The University of Melbourne, Melbourne, Victoria, Australia
}

Correspondence to Professor Linda Denehy, Department of Physiotherapy, The University of Melbourne, Melbourne, Vic 3010, Australia; I.denehy@unimelb.edu.au per day) or control (target $30 \mathrm{~min}$ per day) physical rehabilitation treatment. The primary outcome was health-related quality of life measured using the Physical Component Summary (PCS) of the ShortForm-36 (SF-36) at 6 months. Intentionto-treat analysis revealed no difference between groups, mean (SD) PCS in intervention (37 (12.2)) and control (37 (11.3)) groups, respectively.

Several major strengths of this trial include a study design with high internal validity, notable approaches to minimise bias and embedded process evaluation, and there is transparency around key limitations, namely separation between groups for dose of received intervention, attrition and blinding of key trial personnel. Nonetheless, what can we learn from this trial, where again no difference in primary outcome is reported? Here we examine four key methodological aspects with the lens of hindsight, namely the population studied, timing and dose of intervention and selection of primary outcome.

The current trial identified a target population broadly in keeping with eligibility criteria adopted by previous trials. Randomisation was then stratified by admitting ICU, admission type and level of prehospitalisation independence for activities of daily living. However, high levels of independence were evident across both groups. Notably, the authors did not capture data on comorbidity of enrolled patients; recent analyses have demonstrated that comorbidities, frailty and overall health trajectory may modify the rehabilitation outcomes of ICU survivors. ${ }^{11} 12$ Restricting phenotypical data of enrolled patients in the current study to relatively simple counts of organ dysfunction, and a functional measure that prioritises sarcopaenia without consideration of dynapaenia, is a limitation to interpreting findings. A more detailed profile of the cohort would have been valuable to assist in untangling the influence of these factors on trial endpoints.

The timepoint at which mobilisation is considered 'early' is controversial; trial interventions of active mobilisation or rehabilitation are reported as commencing between 1 and 8 days after ICU admission. ${ }^{13}$ In EPICC, participants receiving the intervention had an average preceding ICU admission of 6 days, with a further 3 days from enrolment to the commencement of physical rehabilitation. ${ }^{10}$ This represents a somewhat later intervention than previous similar trials ${ }^{349}$ and a timepoint that may exceed the point of efficacy in ameliorating the onset of muscle weakness and functional deficit. Extensive data now exist supporting safety and feasibility of mobilisation activities in patients receiving multiple forms of organ support. Our current knowledge directs us towards commencing rehabilitation early following medical stabilisation. However, determining the optimum timepoint requires additional understanding of the underlying biological effects of intensive care unit-acquired weakness including mitochondrial dysfunction, excitationcontraction uncoupling and muscle membrane excitability, ${ }^{14}$ the optimum nutritional regimen ${ }^{15}$ and how to distinguish true fatigue from psychological factors influencing patient motivation to participate.

Determining the necessary 'dose' of rehabilitation intervention is one of the most challenging features of study design. To date, there is no consensus on optimal prescription parameters to direct a personalised training approach. Intervention group patients in EPICC had an a priori target of 90 min of physical rehabilitation, compared with $30 \mathrm{~min}$ in those receiving standard care, the additional duration of session time acting as the 'enhanced intensity'. At the time of study conception, this was a novel approach to address the question of increasing intensity of rehabilitation and attempt separation between groups. Importantly, the authors discriminated between time spent in 'active' physical rehabilitation and non-participatory activities. This is valuable detail, exceeding that previously reported in other trials, which facilitates distinguishing 'true' rehabilitation and avoids unintentional overestimation of intervention duration. In the future, all rehabilitation trials should follow this approach and explicitly report their protocols in line with the Template for Intervention Description and Replication (TIDieR) guidelines. ${ }^{16}$

Nonetheless, the results demonstrate the striking gap between intended and actual delivery of physical rehabilitation; patients received a median (IQR) of 23 (16-28) min and 13 (10-17) min per day in intervention and standard care groups, respectively, and whether there is true separation between groups is unclear. In the intervention group, only $8 \%$ of treatment days included $>45$ min of therapy, 


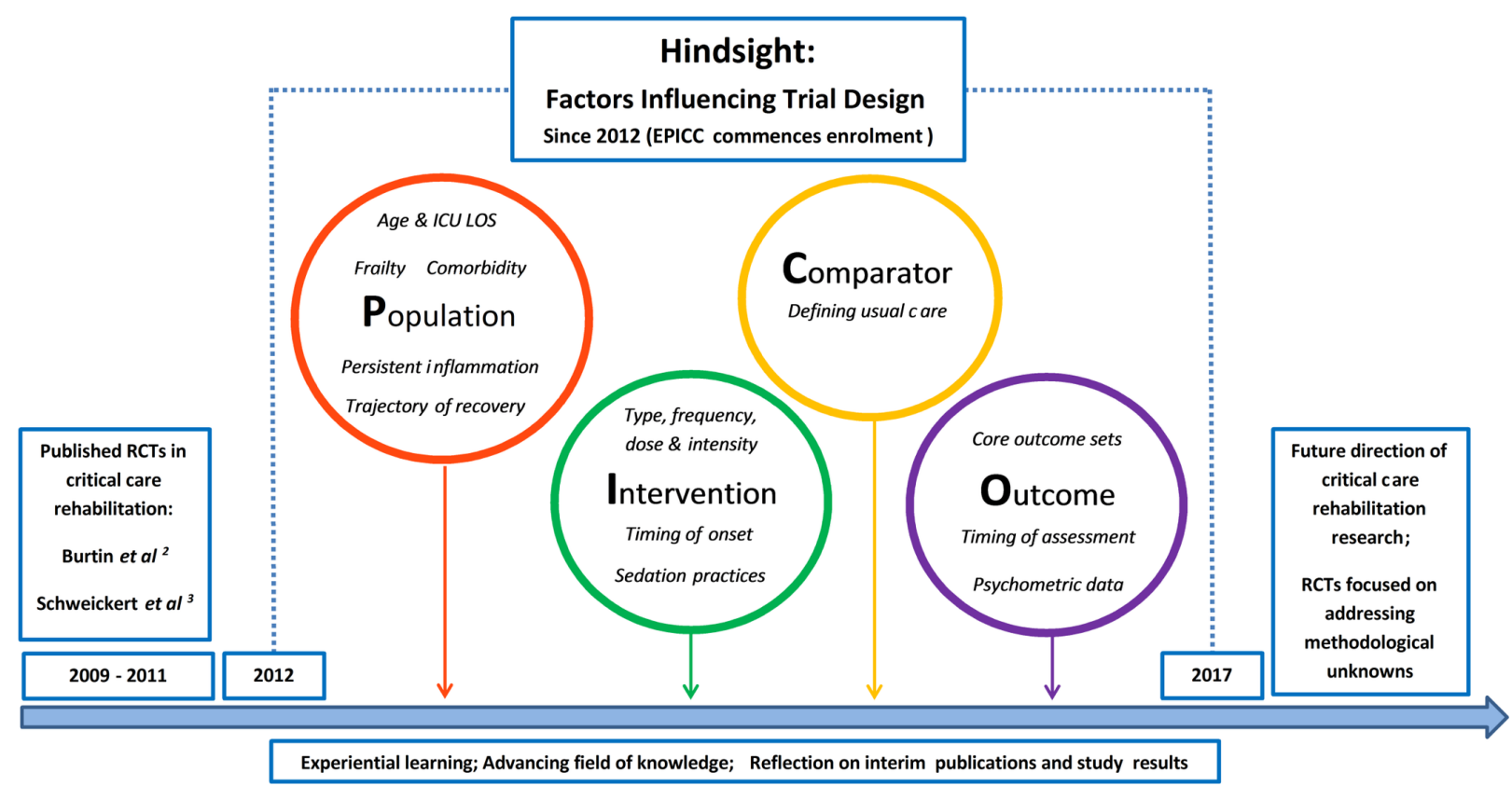

Figure 1 The evolution of critical care rehabilitation trial design. Examples of trials 2009-2011 include physical therapy/exercise-based interventions, excluding electrical muscle stimulation adjuncts. ICU, intensive care unit; LOS, length of stay; RCTs, randomised controlled trials.

and $82 \%$ of treatment days for standard care included $\leq 20 \mathrm{~min}$. That the main limitation to delivery of physical rehabilitation stemmed from observed or reported patient fatigue is not uncommon. ${ }^{17}$ However, detailed reasons for termination of treatment sessions were not collected, and this information should be included in future RCTs to further enhance our understanding of patient responses.

So were the EPICC a priori targets realistic? The authors based their decision making on clinical rationale, centred around defining an intervention that exceeded an existing level of rehabilitation as standard care, inherently more difficult than when standard care is merely the absence of the intervention. Previous trials have typically adopted shorter treatment times, ${ }^{4} 9$ and in keeping with the experience of other authors, ${ }^{9}$ standard care in the current study was greater than the intervention dose in trials from other countries. ${ }^{3}$ As a UK-based study, what is also notable from the current findings is that even under trial conditions, the recommended national guidelines of $45 \mathrm{~min}, 5$ days per week were not able to be achieved. ${ }^{18}$ How therefore can such guidelines infiltrate 'real world' clinical practice where exponential variability in treatment delivery exists?

The primary outcome in EPICC was the SF-36 PCS measuring patient-reported health-related quality of life at 6 months; the SF-36 is a generic instrument, commonly used in this population and a priority measurement instrument for this outcome. ${ }^{19}$ The PCS comprises data from multiple domains of the SF-36, including specific physical function ability for activities such as walking and stair-climbing and broader questions around pain, general health and work-related effort. The current authors found no difference between groups, high variability and little change across time in this outcome. Additionally, there was substantial attrition at 6 months, with only 116 (62\%) of the 308 patients enrolled contributing data (equally from each trial arm) to the final analysis. The authors compare their attrition to other recent trials, ${ }^{4}{ }^{20}$ although the primary outcome was measured at ICU, ${ }^{20}$ and 1 month following hospital, ${ }^{4}$ discharge respectively for these trials where retention was greater. Adopting multiple retention strategies during longitudinal follow-up is paramount in future trials to ensure statistical power of the findings is achieved. ${ }^{21}$ Furthermore, the SF-36 PCS can only be measured in survivors. Accounting for death when measuring patient-reported outcomes over time is critical, especially given that both survival and quality of life are recommended core outcomes for studies of acute respiratory failure survivors. ${ }^{22}$ EPICC did not account for deaths in their analyses and use of differing data management approaches impede comparisons between trials.

SF-36 data from EPICC also contrast to recently published rehabilitation trial data using this outcome measure, ${ }^{7}$ although differing population heterogeneity and attrition, timing of intervention delivery and timing of data acquisition in relation to intervention delivery may be explanatory factors. In the current study, the intervention ceased at ICU discharge with the primary outcome captured at 6 months, with no intervening data collection to explain confounding factors. Trajectories of recovery in SF-36 PCS vary across this and previous studies. In the current trial, recovery was relatively flat for the first 3 months, with a slight increase by 6 months. Similar patterns are evident in the chronic respiratory disease population following pulmonary rehabilitation and may reflect return to an inactive lifestyle or burden of comorbidities on health status. To date, little exploration of the most sensitive component of the SF-36 to use in trials of rehabilitation interventions has been conducted. More research related to outcome timepoints across the trajectory of recovery, and in relation to delivery of interventions, is warranted to inform future trial design.

Since EPICC was commenced in 2012, we have advanced our knowledge around critical care rehabilitation as a complex intervention. Hindsight has allowed us the opportunity to explore the many unanswered methodological questions surrounding our delivery of rehabilitation trials relating to population (establishing patient phenotypes of 'responders'), intervention (determining optimum 'dose'), comparator (accounting for variability in 'usual' care) and outcomes (adopting relevant core outcome sets) and, consequently, the future direction of investigation (figure 1). This knowledge was unbeknownst to Wright et al when designing their trial pre-2012 and 
is important to remember in the interpretation of their findings. However, a cautionary lesson is learnt from this and previous trials of physical rehabilitation in critical illness. Further RCTs are not warranted without at least addressing one or more of these unanswered questions, in order that we ascertain the right intervention, delivered in the right dose and to the right patient. This is after all our raison d'etre.

Acknowledgements The authors would like to acknowledge the creative input of Jennifer Jones (Physiotherapy Department, School of Health Sciences, The University of Melbourne) for her contribution in the development of the graphic featuring in figure 1.

Contributors Both authors contributed equally to the design, drafting and revision of this manuscript and agreed the final version for submission.

Funding $B C$ is funded by an National Institute for Health Research (NIHR) Postdoctoral Fellowship (2015-08-015) and is supported by the NIHR Biomedical Research Centre based at Guy's and St Thomas' NHS Foundation Trust and King's College London.

Disclaimer The views expressed are those of the authors and are not necessarily those of the NHS, the NIHR or the Department of Health.

Competing interests None declared.

Provenance and peer review Commissioned; externally peer reviewed.

(c) Article author(s) (or their employer(s) unless otherwise stated in the text of the article) 2018. All rights reserved. No commercial use is permitted unless otherwise expressly granted.

\section{D) Check for updates}

To cite Connolly B, Denehy L. Thorax 2018:73:203-205.

Published Online First 14 November 2017

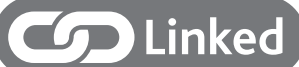

- http://dx.doi.org/10.1136/thoraxjnl-2016-209858

Thorax 2018;73:203-205

doi:10.1136/thoraxinl-2017-210588

\section{REFERENCES}

1 Collins English Dictionary. London: Harper Collins Publishers. https://www.collinsdictionary.com/ dictionary/english/. (accessed 06Jul 2017).

2 Burtin C, Clerckx B, Robbeets C, et al. Early exercise in critically ill patients enhances short-term functional recovery. Crit Care Med 2009;37:2499-505.

3 Schweickert WD, Pohlman MC, Pohlman AS, et al. Early physical and occupational therapy in mechanically ventilated, critically ill patients: a randomised controlled trial. Lancet 2009;373:1874-82.

4 Moss M, Nordon-Craft A, Malone D, et al. A randomized trial of an intensive physical therapy program for patients with acute respiratory failure. $A m$ J Respir Crit Care Med 2016:193:1101-10.

5 Morris PE, Berry MJ, Files DC, et al. Standardized rehabilitation and hospital length of stay among patients with acute respiratory failure: a randomized clinical trial. JAMA 2016:315:2694-702.

6 Walsh TS, Salisbury LG, Merriweather JL, et al. Increased hospital-based physical rehabilitation and information provision after intensive care unit discharge: the RECOVER randomized clinical trial. JAMA Intern Med 2015:175:901-10.

7 McDowell K, O'Neill B, Blackwood B, et al. Effectiveness of an exercise programme on physical function in patients discharged from hospital following critical illness: a randomised controlled trial (the REVIVE trial). Thorax 2017;72:594.1-5

8 Connolly B, Salisbury L, O'Neill B, et al. Exercise rehabilitation following intensive care unit discharge for recovery from critical illness. Cochrane Database Syst Rev 2015:CD008632.

9 Denehy L, Skinner EH, Edbrooke L, et al. Exercise rehabilitation for patients with critical illness: a randomized controlled trial with 12 months of followup. Crit Care 2013:17:R156

10 Wright SE, Thomas K, Watson G, et al. Intensive versus standard physical rehabilitation therapy in the critically ill (EPICC): a multicentre, parallel-group, randomised controlled trial. Thorax 2018;73:213-21.
11 Puthucheary ZA, Denehy L. Exercise interventions in critical illness survivors: understanding inclusion and stratification criteria. Am J Respir Crit Care Med 2015:191:1464-7.

12 Ferrante LE, Pisani MA, Murphy TE, et al. Factors associated with functional recovery among older intensive care unit survivors. Am J Respir Crit Care Med 2016:194:299-307.

13 Tipping CJ, Harrold M, Holland A, et al. The effects of active mobilisation and rehabilitation in ICU on mortality and function: a systematic review. Intensive Care Med 2017;43:171-83.

14 Batt J, Mathur S, Katzberg HD. Mechanism of ICUacquired weakness: muscle contractility in critical illness. Intensive Care Med 2017;43:584-6.

15 Arabi YM, Casaer MP, Chapman M, et al. The intensive care medicine research agenda in nutrition and metabolism. Intensive Care Med 2017:18:1239-56

16 Hoffmann TC, Glasziou PP, Boutron I, et al. Better reporting of interventions: template for intervention description and replication (TIDieR) checklist and guide. BMJ 2014;348:g1687.

17 Berney S, Haines K, Skinner EH, et al. Safety and feasibility of an exercise prescription approach to rehabilitation across the continuum of care for survivors of critical illness. Phys Ther 2012:92:1524-35.

18 Intensive Care Society Core Standards for Intensive Care Units. Intensive Care Society. 2013 http://www. ics.ac.uk/ICS/Education/Guidelines__Standards/ICS/ guidelines-andstandards. aspx?hkey=4ed20a1 c-1ff846e0-b48e-732f1f4a90e2 (accessed 14 Dec 2014).

19 Needham DM, Sepulveda KA, Dinglas VD, et al. Core outcome measures for clinical research in acute respiratory failure survivors. an international modified delphi consensus study. Am J Respir Crit Care Med 2017:196:1122-30

20 Schaller SJ, Anstey M, Blobner M, et al. Early, goal-directed mobilisation in the surgical intensive care unit: a randomised controlled trial. Lancet 2016:388:1377-88

21 Abshire M, Dinglas VD, Cajita Ml, et al. Participant retention practices in longitudinal clinical research studies with high retention rates. BMC Med Res Methodol 2017:17:30

22 Turnbull AE, Sepulveda KA, Dinglas VD, et al. Core domains for clinical research in acute respiratory failure survivors: an international modified delphi consensus study. Crit Care Med 2017:45:1001-10. 